\title{
Automated Spinal Cord Monitoring for Spinal Surgery
}

\author{
M. Imai, MS, Y. Harada, MD, Y. Atsuta, MD, Y. Takemitsu, MD, T. \\ Iwahara, MD \\ Department of Orthopaedic Surgery, Asahikawa Medical College, Asahikawa, \\ Fapan.
}

\begin{abstract}
Summary
A microcomputer-based automated spinal cord monitoring system was developed and 56 spinal operations have been carried out with it since 1986. The system comprises an electrodiagnostic system (Dantec 2000M), an FM tape recorder, a channel selector, an electrical relay, a Modem and a microcomputer which controls all of the elements. The program allowed for automated recording and analysis of evoked potentials during spinal surgery and alarm signals were generated according to the criteria of amplitude decrease and latency delay. It was demonstrated that automated spinal cord monitoring in the operating room was performed easily, reliably and safely allowing the surgeon to react quickly to possible compromise of the spinal cord. The design and usefulness of the system is described in this report.
\end{abstract}

Key words: Spinal cord evoked potentials; Alarm system; Automated monitoring; Remote control; Microcomputer.

Recent studies have reported that intra-operative monitoring of spinal cord integrity using spinal cord evoked potentials as well as somato-sensory evoked potentials is useful in preventing neurological complications (Engler, 1978; Tamaki, 1981; Ryan, 1983; Jones, 1983). In the past, a specialist had to repeat meticulous operations of electrodiagnostic instruments and analyse recorded data many times to monitor evoked potentials during surgery. Thus, for intraoperative monitoring to be used widely and easily, an automated system is necessary (Nash, 1977; Harada, 1983). In this study, the development of a fully automated spinal cord monitoring system was attempted. This microcomputerbased system made it possible to automatically carry out serial operations of electrostimulation, averaging of evoked potentials, storing the most recent data onto a floppy disk and/or FM tape, and the generation of alarm signals based on comparison of the amplitudes and latencies of the most recent potentials 


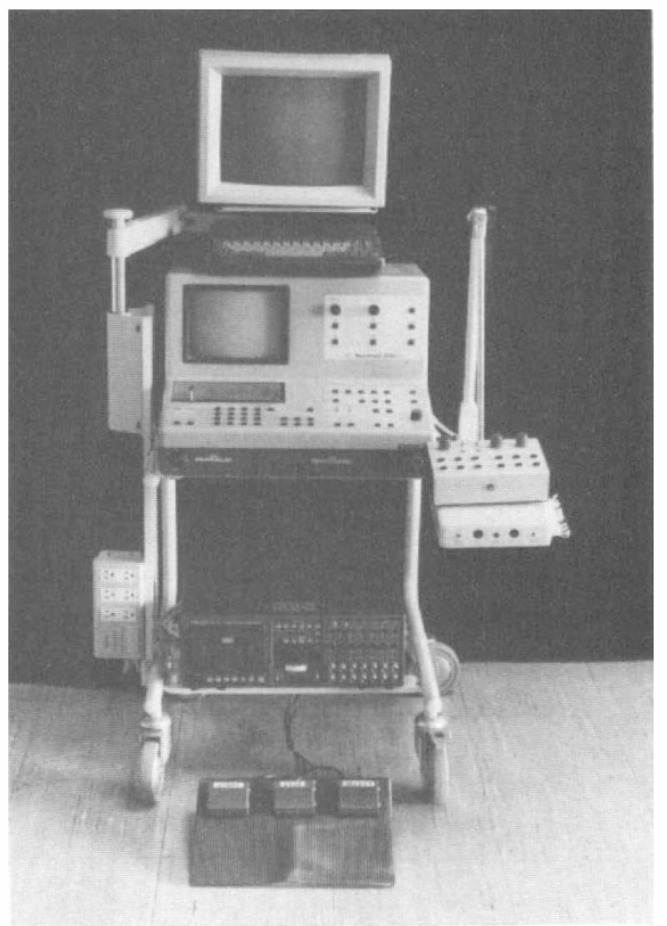

Figure 1 The system consists of an electrodiagnostic system (Dantec Neuromatic 2000 M), an FM tape recorder, a channel selector, an electronic relay, a Modem and a microcomputer (NEC LV21).

with those of a control. It was also necessary to control the power lines of an electrocautery and lines to the patient for stimulation and recording by computer in order to maintain reliability of the system and the patient's safety in the operating room. This system allows the surgeon to send a start signal by simply tripping a foot switch. He can then quickly see the interpreted data. Preliminary results have been reported (Imai, 1986, 1987).

\section{Materials and methods}

The system consists of an electrodiagnostic system (Dantec Neuromatic 2000 $\mathrm{M})$, an FM tape recorder, a channel selector, an electronic relay, a Modem*, and a microcomputer (NEC LV 21) which controls all the elements (Fig. 1). The channel selector was equipped to perform automatic connection and shutting off between the patient's electrodes, the electrostimulator, and the amplifiers. The electrocautery is automatically shut down by a relay switch while the monitoring procedure is carried out.

Programs were written in $\mathrm{C}$ and assembly language. Amplitudes and peak latencies of evoked potential were automatically measured and compared with those of a control potential (Fig. 2). Further processing of acquired data such

* Modem: a device that converts data to a form that can be transmitted, as by telephone, to dataprocessing equipment, where a similar device reconverts it. 


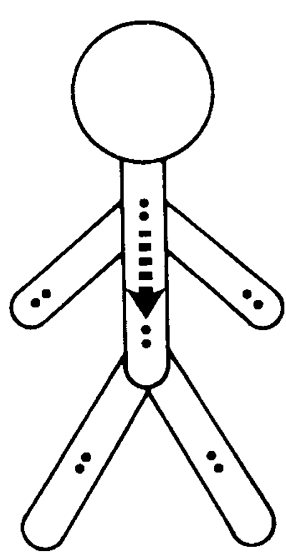

$\begin{array}{cccccc}\text { Peak } & 1 & 2 & 3 & 4 & 5 \\ \text { Amplitude (\%) } & & 68.5 & 66.4 & 79.1 & \\ \text { Latency (mS) } & 0.21 & 0.45 & 0.66 & 0.74 & 0.59\end{array}$

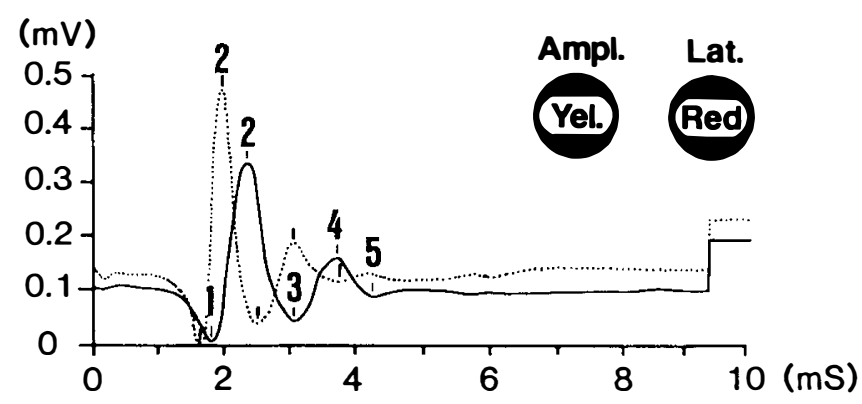

Figure 2 An example display on the microcomputer screen during automated monitoring. Peaks of the latest potential (solid line) are detected, and values, such as percentage of amplitude and delay of latency, are also shown, compared with the control potential (dotted line) (negatively upward).

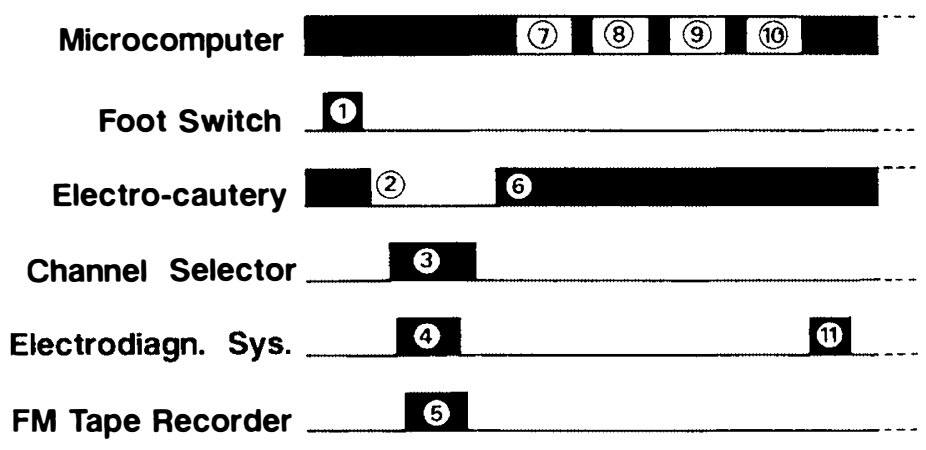

Figure 3 Timing chart of the automated spinal cord monitoring.

as time course, addition, subtraction, smoothing and statistical analysis were also available.

The intra-operative automatic monitoring was carried out in the following order (Fig. 3):

1. The surgeon steps on the foot switch to send a start signal to the computer. The computer is also able to generate starting signals automatically at a preset interval.

2. The power of electrocautery is cut off by a relay switch.

3. The channel selector chooses between stimulating and recording electrodes, and connects them to the stimulator and amplifier of the electrodiagnostic system.

4. The evoked potential is averaged and smoothed to eliminate signal noise.

5. The FM tape recorder is driven to record raw signals, surgeon's voice, ECGs and arterial pressure for later analysis.

6. Electrode lines to the patient are switched off and power to the electrocautery is restored. 

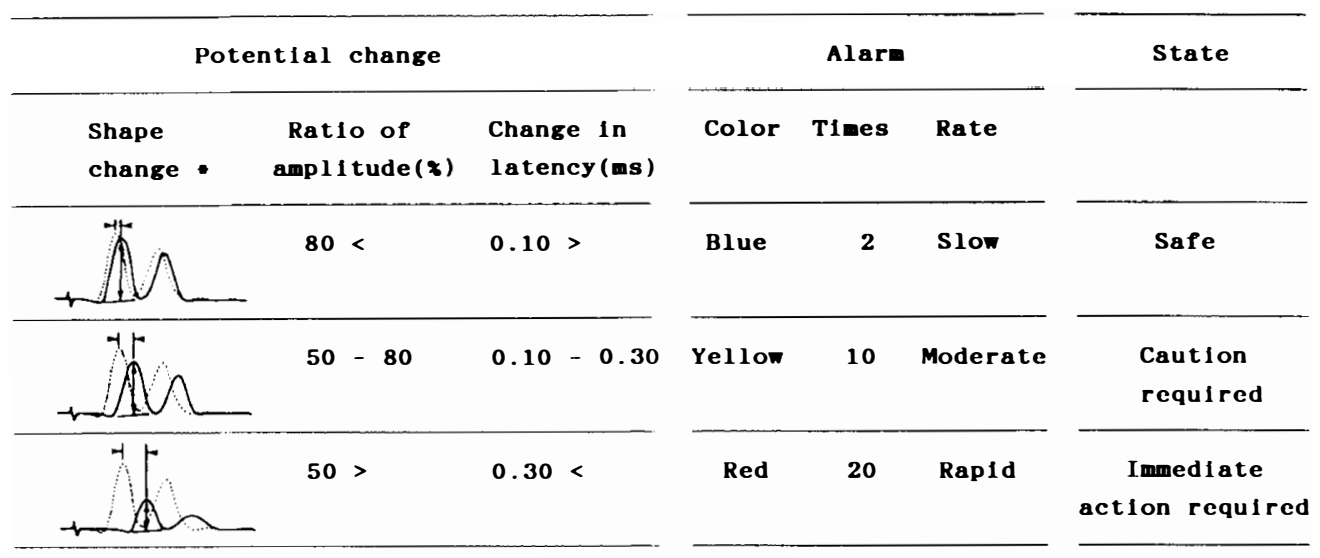

Figure 4 Criteria of alarm signals. Solid line: latest potential, dotted line: control.

7. The averaged potential is acquired by microcomputer.

8. Amplitudes and latencies of detected peaks are measured and compared with control.

9. Alarm signals are generated to the surgeon according to the criteria chosen.

10. Data is stored on the floppy disc.

11. A hardcopy of the potential is made. Data of amplitude and latency are also described.

Alarm signals are given by means of a flashing screen display and a buzzer from the microcomputer. Three signal patterns were prepared to inform the surgeon of the grade of alteration in the evoked potentials according to the following criteria (Fig. 4):

1. Safe range (amplitude over $80 \%$, latency delay within $0 \cdot 1 \mathrm{~ms}$ ); blue flashing screen-buzzer sounded twice slowly.

2. Caution range (amplitude $50-80 \%$, latency delay $0 \cdot 1-0.3 \mathrm{~ms}$ ); yellow flashing screen-buzzer 10 times at moderate speed.

3. Danger range requiring immediate action (amplitude below $50 \%$, latency delay over $0.3 \mathrm{~ms}$ ); red flashing screen-buzzer 20 times rapidly.

Since 1986, 56 spinal surgeries have been performed using this automated spinal cord monitoring system. They include 35 patients with incomplete paralysis due to cervical and/or thoracic spinal canal stenosis, 12 spinal deformities, 4 spinal tumours and 5 traumatic spinal injuries. In most of the cases, spinal cord potentials evoked by electrostimulation of spinal cord or peripheral nerves were used.

\section{Results}

In all cases, automated spinal cord monitoring was successfully performed. The system was used to perform monitoring functions an average of 21.3 times per surgery. Interpreted data was available to the surgeon 20 seconds after the starting signal from the foot switch. 


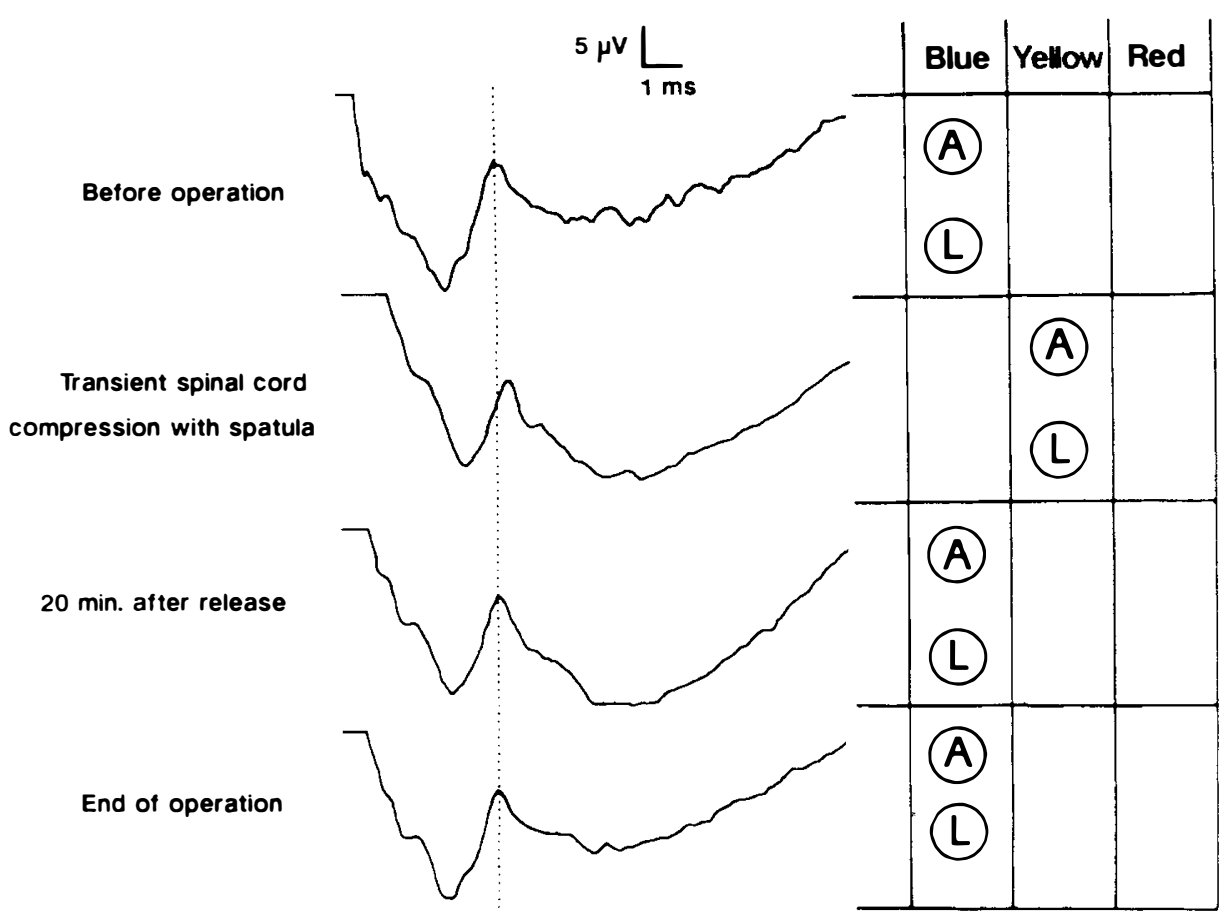

Figure 5 Intra-operative potential (stim.:T12, record.:T7) change and alarms during decompressive surgery for myelopathy due to ossification of the posterior longitudinar ligaments at T9, T10, T11 levels (circle A; Amplitude, circle L; Latency).

None of the cases showed neurological complications after the surgery. In 2 cases of corrective surgery for scoliosis, a yellow alarm due to a delay in the latency was generated. In both cases, after confirming the delay was not accompanied by a decrease in amplitude, surgery was continued. Despite low amplitude of evoked potentials in the cases with incomplete paralysis, monitoring was possible. In 3 cases, a false alarm signal was generated by the dislocation of a recording electrode. In the following case, the system played a notably important role.

A 58-year-old male patient had a decompression of the thoracic spine from incomplete paralysis due to ossification of the posterior longitudinal ligament. The spinal cord evoked potential was used for the automated monitoring. A yellow alarm was generated when the decompression from T9 to T10 was completed (Fig. 5). At that time, it was found that a spatula was slightly compressing the spinal cord. After observing the recovery of the potential, T11 decompression was completed. No neurological complication was observed after surgery.

A comparison of the 3 signals generated during automatic monitoring is shown in Figure 6. Percentages of each of the 3 signals were nearly equal in both amplitude and latency. 


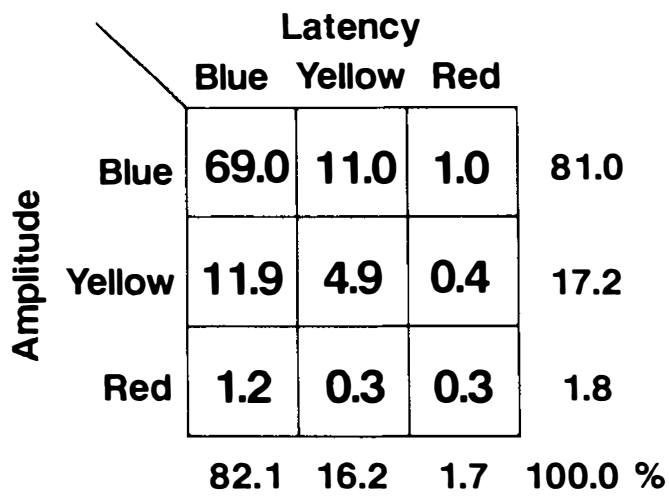

Figure 6 Comparison of 3 signals generated by automatic monitoring.

\section{Discussion}

It has been reported that iatrogenic neurological complications may be caused by corrective surgery for severe spinal deformity, reconstructive surgery for injured spine, as well as decompressive surgery for degenerative spinal diseases, ossification of the posterior longitudinal ligament and spinal tumours (Ponte, 1973; MacEwen, 1975). According to the morbidity reports of Scoliosis Research Society (SRS) 1987, the incidence of neurological injury during surgery was reported to be $1.84 \%$ (SRS 1987). In Japan, spinal cord evoked potentials have been applied for the monitoring of the integrity since 1972 (Kurokawa, 1972; Tamaki, 1972). Although a partly automated monitoring system has been reported (Boston 1984; Micheli-Tzanakou 1984; Gopalan 1986; Friedman 1987), the system described in this report has fully automated operations of all instruments including an alarm to the surgeon.

In the development of the system, the emphasis has been placed on patient safety. A photo-coupled module used in this system shuts off electrical leakage. Also, the lines connecting the electrical equipment and the patient were disconnected while not in use to prevent microshock. A program capable of recovering the latest system operations and data was prepared to protect the system from the system down due to power supply voltage drop, electrical noise, or power breakdown. Three types of alarm signals were prepared according to the grade of change in an amplitude and a latency. The alarm with a flashing display and buzzer allowed the surgeon to recognise the interpreted result in a moment. Although the criteria for each alarm in relation to spike amplitude is still controversial, we chose a $50 \%$ decrease from the control as a dangerous limit (red alarm) according to Atsuta (1987), Iwahara (1987). However, even in the yellow alarm range, it would be advisable for the surgeon to recheck the procedures carefully.

Slight latency delay in the yellow alarm range without amplitude decrease was observed in two scoliosis patients. It was believed to be caused by an elongation of the distance between the stimulating and the recording electrodes. Further attention would have been necessary had the latency delay been accompanied by an amplitude decrease.

In Figure 6, the majority of the results were acceptable, however, the cases of 
amplitude-blue and latency-red signals, and amplitude-red and latency-blue signals were unacceptable. The former signals were probably due to dislocation of the recording electrode. In such cases, it was necessary to restart the monitoring using a newly obtained evoked potential as a reset control. The later signals may be caused by an averaging of contaminated potentials, it was necessary to isolate recording conditions from electrical or mechanical noise.

A still unresolved issue is whether it is sufficient to check the amplitude and latency of only the first potential to generate an alarm signal. It may be necessary to choose the most important element in the evoked potential according to the nature of the potential adopted and the condition of the spinal cord as well as the operating method, in this system criteria of each signal is changeable.

In addition to spinal surgery, this system is applicable to a variety of surgical procedures in which neurological complications may be elicited (Stone, 1985; Kaplan, 1986; Lee, 1987; Ariyama, 1989). Furthermore, since this system is applicable not only to the monitoring of surgery but also to later analysis of the data, it will contribute to improving the clinical results of surgical operations.

\section{Acknowledgement}

We wish to acknowledge valuable discussions with Mr Y. Yamada, I. Iijima, E. Yamazaki and their substantial efforts in implementing the system. We would like to thank $\mathrm{Mr}$ Michael Webber for many suggestions in writing this paper.

This study was supported in part by Ministry of Education and Culture of Japan Grant in Aid for Scientific Research (B) 60480334.

\section{References}

Ariyama H, Atsuta Y, ANdo M, Goto E. 1989 Sciatic nerve monitoring system during hip arthroplasty. Clinical Orthopaedic Surgery (in press).

ATsUTA Y, HARADA Y, IwAHARA T, et al. 1987 Relationship between locomotor function and spinal cord evoked potential during acute spinal cord compression. Fournal of Electrodiagnosis of the Spinal Cord 10(1):5-8.

Boston JR, DenEAult LG 1984 Sensory evoked potentials: A system for clinical testing and patient monitoring. International fournal of Clinical Monitoring and Computing 1(1):13-19.

EDWARDS BN, Tullos HS, NOBLE PC 1987 Contributory factors and etiology of sciatic nerve palsy in total hip arthroplasty. Clinical Orthopaedics and Related Research 218:136-141.

ENGLER GL, SPIELHOLz NI, BERNHARD WN, et al. 1978 Somatosensory evoked potentials during harrington instrumentation for scoliosis. The fournal of Bone and foint Surgery 60-A(4):528532.

Friedman WA, CURRAN MT 1987 Somatosensory Evoked Potentials after Sequential Extremity Stimulation:A New Method for Improved Monitoring Accuracy. Neurosurgery 21(5):755-758.

Gopalan R, PARKer PA, SCOTt RN 1986 Microprocessor-based system for Monitoring spinal evoked potentials during surgery. IEEE Transactions Biomedical Engineering 33(10):982-985.

HARADA Y 1983 Study on experimental spinal cord injuries by distraction using spinal cord evoked potentials. Fournal of the fapanese Orthopaedic Associatia 57:685-701.

Imai M, HARADA Y, Atsuta Y, et al. 1986 Microcomputer-based system for automated measurements and analysis of spinal cord evoked potentials. Proceedings of 6 th Joint Conference on Medical Informatics, pp 207-208.

IMAi M, HARADA Y, AtSUTA Y, et al. 1987 Automated spinal cord monitoring system using a micro-computer. Fournal of Electrodiagnosis of the Spinal Cord 10(1):75-78.

IWAHARA T, HARADA Y, ATSUTA Y, et al. 1987 Relation between spinal cord evoked potential and spinal cord blood flow under spinal cord distraction. Fournal of Electrodiagnosis of the Spinal Cord 10(1):83-86.

Johanson NA, PellicCi PM, Tsairis P, et al. 1983 Nerve injury in total hip arthroplasty. Clinical Orthopaedics and Related Research 179: 214-222.

JoNES SJ, EDGAR MA, RANSFORD AO, et al. 1983 A system for the electrophysiological monitoring of the spinal cord during operations for scoliosis. Fournal of Bone and foint Surgery 65b(2): 134-139. 
KAPLAN BJ, et al. 1986 Somatosensory evoked potential monitoring of spinal cord ischemia during aortic operations. Neurosurgery 19:82-90.

KUROKAWA T 1972 Spinal cord action potentials evoked by epidural stimulation of cord-A report of human and animal record. Fapanese fournal of Electroencephalography, Electromyography and Clinical Neurophysiology 1:64.

LeE K, Hirabayashi S, Hoshino Y, et al. 1987 Spinal cord monitoring during operations on aortic aneurysm. Fournal of Electrodiagnosis of the Spinal Cord 10(1):95-98.

Micheli-Tzanakou J, Ingeholm J Jr, Haber L 1984 Collection and storage of data for evoked potential studies: A database. Computer Programs in Biomedicine 18:51-60.

NASH CL, LoRig RA, SchatzINGER LA, et al. 1977 Spinal cord monitoring during operative treatment of the spine. Clinical Orthopaedics and Related Research 126:100-105.

PONTE A 1973 Postoperative paraplegia due to hypercorrection of Scoliosis Research Society, Gothenburg, Sweden, 17-21 September.

RYAN TP, BRITT RH 1983 Intraoperative Cortical and Spinal SEP Monitoring. Proceedings of the Annual Conference IEEE Engineering Medical Biological Society 5:225-230.

SCOLIOSIS RESEARCH SOCIETY 1987 Morbidity and Mortality Report.

STONE RG, WEEKs LE, HAJDU M, et al. 1985 Evaluation of sciatic nerve compromise during total hip arthroplasty. Clinical Orthopaedics and Related Research 201:26-31.

TAMAKI T, YAMASHITA T, KOBAYASHI H, et al. 1972 Spinal cord monitoring. fapanese fournal of Electroencephalography, Electromyography and Clinical Neurophysiology 1:196.

TAMAKI T, Tsuji $H$, INOUE $S 1981$ The prevention of iatrogenic spinal cord injury utilizing the evoked spinal cord potential. International Orthopaedics (SICOT) 4:313-317.

Weber ER, DAube JR, Coventry MB, et al. 1976 Peripheral neuropathies associated with total hip arthroplasty. The fournal of Bone and foint Surgery 58-A(1):66-69. 